Published in final edited form as:

Prog Brain Res. 2012 ; 195: 91-102. doi:10.1016/B978-0-444-53860-4.00005-2.

\title{
The evolution of neocortex in primates
}

\author{
Jon H. Kaas \\ Department of Psychology, Vanderbilt University, Nashville, TN, USA
}

\begin{abstract}
We can learn about the evolution of neocortex in primates through comparative studies of cortical organization in primates and those mammals that are the closest living relatives of primates, in conjunction with brain features revealed by the skull endocasts of fossil archaic primates. Such studies suggest that early primates had acquired a number of features of neocortex that now distinguish modern primates. Most notably, early primates had an array of new visual areas, and those visual areas widely shared with other mammals had been modified. Posterior parietal cortex was greatly expanded with sensorimotor modules for reaching, grasping, and personal defense. Motor cortex had become more specialized for hand use, and the functions of primary motor cortex were enhanced by the addition and development of premotor and cingulate motor areas. Cortical architecture became more varied, and cortical neuron populations became denser overall than in nonprimate ancestors. Primary visual cortex had the densest population of neurons, and this became more pronounced in the anthropoid radiation. Within the primate clade, considerable variability in cortical size, numbers of areas, and architecture evolved.
\end{abstract}

\section{Keywords}

prosimians; tarsiers; anthropoids; sensory cortex; motor cortex

\section{Introduction}

This review focuses on the areal and structural organization of neocortex in primates. This is an especially challenging topic to review, as primates constitute a highly diversified taxon consisting of 14 families and at least 350 extant species. Primates emerged as a distinct line of evolution around 80million years ago (Martin, 2004) and diversified within three early branches leading to present-day prosimians, tarsiers, and anthropoids (monkeys, apes, and humans). Primates have adapted to a wide range of environments, allowing them to vary in size 5000-fold from the mouse lemur at $40 \mathrm{~g}$ to the male gorilla, sometimes over $200 \mathrm{~kg}$. Neocortex is a major part of the brain of all primates, especially so in humans where cerebral cortex occupies $80 \%$ of the brain mass and contains 16 billion neurons (Avzevedo et al., 2009). Despite a huge variability in absolute brain size, and the size of neocortex, a characteristic pattern of areal organization has been found in all studied primates. Numbers of cortical areas, connection patterns, and structural and functional organization of areas all vary. Yet, a set of cortical areas exists in all primates, and some of these areas appear to be unique with primates. Here, we first consider the organization of neocortex in a prosimian primate that has been extensively studied, the African galago, compare the organization of neocortex in primates with that of the close relatives of primates to suggest how the primate pattern might have evolved, and briefly considering some of the major variations in cortical organization and structure that we see across the primate radiation.

Copyright ( 92012 Elsevier B.V. All rights reserved.

"Corresponding author: Tel.: +1-615-3223029; Fax: +1-615-3438449, jon.h.kaas@ vanderbilt.edu. 


\section{Cortical organization in prosimian galagos: Comparisons with other primates}

Prosimian primates have been of special value to those interested in the evolution of primates because they resemble the early ancestors of primates more closely than members of the tarsier and anthropoid radiations. However, early primates were small, had smaller brains relative to body size, and depended less on vision and more on olfaction than modern prosimians (Martin, 2004; Ni et al., 2003). While the prosimian radiation of galagos, lorises, and lemurs includes a range of adaptations, it is presently unclear how much prosimians vary in cortical organization, as only galagos have been well studied.

In galagos, neocortex is subdivided into a number of areas that are also recognized in other primates. These areas are shown on a flattened surface view of the neocortex so that the relationship of proposed cortical areas can be seen, including areas not apparent on a dorsolateral view of the brain as they are hidden on the ventral and medial surfaces of the brain, and in the few fissures found in galago cortex (Fig. 1). Here, we describe some of the major functional divisions of neocortex in galagos and discuss those divisions in relation to what is known in other primates and nonprimate relatives of primates.

\section{Visual cortex}

The full extent of visual cortex and the total number of visual areas is not known in galagos, as it can be difficult to define cortical areas, and even in the more extensively studied macaques, there are uncertainties. However, a collection of visual areas has been defined (Fig. 1), and these areas also exist in other primates. The primary and secondary visual areas, V1 and V2, exist in nearly all mammals, but these areas have specialized features in primates. Thus, galagos (Rosa et al., 1997) and other primates have greatly expanded representations of central vision in V1 and other visual areas. In addition, V1 has sublayers of layer 4 that are different from those in other taxa. The inner half of layer 4 receives inputs from the parvocellular layers of the lateral geniculate nucleus and these inputs are especially important in detailed object vision, while the outer half of layer 4 receives inputs from the magnocellular layers, and these inputs are important in detecting motion and change (Casagrande and Kaas, 1994). The superficial layers of cortex express a dot-like distribution of functional modules, the so-called blobs, which may be important in color vision (Casagrande and Kaas, 1994; Preuss and Kaas, 1996). Because these features are not found in the nonprimate members of the Euarchontoglire clade (rabbits, rodents, flying lemurs, tree shrews, and primates), they must have evolved in the line leading to early primates (Kaas, 2005). However, both primates and tree shrews have orderly arrangements of orientationselective neurons in V1, while these neurons are randomly distributed in rodents (Van Hooser et al., 2006). Thus, columns of orientation-selective neurons in V1 likely evolved in the common ancestors of the tree shrews and primates.

In almost all studied mammals, V1 is bordered along most of its perimeter by the second visual area, V2 (Rosa and Krubitzer, 1999). In anthropoid primates, V2 is uniquely subdivided into repeating sets of three types of band-like modules that cross the width of the $\mathrm{V} 2$ belt. These bands can be revealed by stains for cytochrome oxidase (CO) or myelin and have been characterized as CO-dense thick or thin bands, or CO-light (pale) bands. Each type of band has different inputs from modules and layers in V1 and outputs to other cortical areas, and neurons of different functional properties (Casagrande and Kaas, 1994). Studies of connection patterns in prosimian primates suggest that V2 is subdivided into the same three types of modules in these primates as well, but the V2 modules are only weakly apparent in CO or myelin preparations (Collins et al., 2001; Preuss et al., 1993). Rodents and tree shrews do not have this type of modular organization in V2. Thus, the V2 bands evolved with the first primates and became histologically more distinct in anthropoid primates. 
V3 is another visual area common to all primates. This conclusion was in question until recently when connection patterns with V1 were used to clearly identify V3 in prosimian galagos (Lyon and Kaas, 2002a), and new and old world monkeys (Lyon and Kaas, $2002 \mathrm{~b}, \mathrm{c})$. As of now, there is no compelling evidence for V3 in rodents, rabbits, or tree shrews, the close relatives of primates, so V3 may have emerged with primates. If so, the V3 described in cats and other carnivores evolved independently.

In all primates studied, $\mathrm{V} 1$ projects to a densely myelinated area in the middle of the upper temporal lobe, the middle temporal (MT) visual area, where neurons are sensitive to stimulus orientation and direction of motion (Kaskan et al., 2010). Because of its histological distinctiveness (Allman and Kaas, 1971), MT has been histologically identified in humans (Tootell and Taylor, 1995) well before it was possible by imaging (fMRI), and in tarsiers (Wong et al., 2010), which are unavailable for experimental study. An area highly similar to MT has not been identified in tree shrews, rodents, or rabbits, raising the likelihood that MT is new with primates, or that MT is an area that has been so modified in primates that homologues of MT are not recognizable as MT in other members of the Euarchontoglire clade (Kaas and Preuss, 1993).

Finally, all primates appear to have visual areas, termed here the dorsolateral visual area, DL (Allman and Kaas, 1974), and the dorsomedial visual area, DM (Allman and Kaas, 1975). These areas have been less well defined than V1, V2, and MT, and their boundaries have been adjusted by various investigators. DL is also termed V4 and DM also termed V3a. Together with MST, FST, and MTc, areas associated with MT (Kaas and Morel, 1993), DL, and DM are likely components of visual cortex in all primates (again with no certain homologues in other mammals). Prostriata (Fig. 1) is a limbic visual area that is found in most mammals (Rosa and Krubitzer, 1999). Other visual areas have been proposed, but these areas have not been well defined in a range of primate taxa.

\section{Auditory cortex}

Auditory cortex has been less extensively studied in primates than visual cortex, and little is known about auditory cortex organization in galagos. The standard model for the early stages of processing in auditory cortex of primates now includes a core of three primary or primary-like areas, A1 (the primary area), R (the rostral area), and RT (the rostrotemporal area), surrounded by a belt of eight secondary areas, bordered laterally by a parabelt, a third level of cortical processing of two divisions (Kaas and Hackett, 2000). This model appears to apply to old world macaque monkeys and new world monkeys (Kaas, 2011), and recent imaging and histological evidence from humans is consistent with the model (Sweet et al., 2005; Woods et al., 2010). In a review, Brugge (1982) provided evidence for two core auditory areas in galagos, $\mathrm{R}$ and $\mathrm{A} 1$, and one of the most distinctive of the belt areas (CM), so it is reasonable to conclude that all primates share at least two core areas and some belt areas of auditory cortex. Areas A1, R, some or all of the belt, and perhaps the parabelt are likely common to all primates (Fig. 1). Since all or nearly all mammals have a primary auditory area or areas, as well as secondary fields (Kaas, 2011), some of the areas proposed for primates probably were retained from early nonprimate ancestors. However, the common existence of more than one primary area in mammals makes the identification of homologous primary areas difficult.

In macaque monkeys, it is clear that larger regions of cortex are involved in processing auditory signals (Poremba and Mishkin, 2007), and this is the case for humans as well where specializations for language occur (Scott and Johnsrude, 2003). Thus, it seems likely that major differences exist in the number and organization of higher auditory areas across primate taxa. 


\section{Somatosensory cortex}

The somatosensory cortex of galagos includes a primary area, $3 \mathrm{~b}$, as in other primates, a narrow area $3 \mathrm{a}$ with proprioceptive inputs along the rostral border of area $3 \mathrm{~b}$, and a band of cortex along the caudal border of area $3 \mathrm{~b}$ with inputs from area $3 \mathrm{~b}$ that resembles area 1 or area 1 plus area 2 of anthropoid primates (Fig. 1). Area $3 \mathrm{~b}$ is homologous to $\mathrm{S} 1$ as defined in most nonprimate mammals (Kaas, 1983), and area 3a, with inputs from a proprioceptive nucleus in the thalamus, the ventroposterior superior nucleus in primates, is homologous to a dysgranular representation of muscle and joint receptors along the rostral border of S1 that has been described in rats, cats, raccoons, and other mammals (Kaas, 2007). The band of somatosensory cortex just caudal to area $3 \mathrm{~b}$ of galagos does not respond well to somatosensory stimuli in anesthetized galagos but is in the position of area 1, or area 1 plus area 2 , of anthropoid primates. In all primates, area $3 \mathrm{~b}$ contains a systematic representation of cutaneous receptors of the contralateral half of the body, in addition to representations of the ipsilateral teeth and tongue (Kaas et al., 2006). Thus, both the contralateral and the ipsilateral tongue and teeth are represented in area $3 \mathrm{~b}$ of each hemisphere. Area $3 \mathrm{~b}$ consistently represents the contralateral body surface from foot to face in a mediolateral sequence across cortex (Fig. 1). In primates, a large portion of the representation is devoted to the glabrous hand, and a larger portion is devoted to the face, teeth, and tongue. In galagos and most monkeys, little cortex is used to represent the tail, but the new world monkeys that have evolved a prehensile tail with a ventral pad of glabrous sensory skin have also evolved a large representation of the tail in medial area $3 b$ (Felleman et al., 1983). In anthropoid primates, area 1, the strip of cortex immediately caudal to area $3 \mathrm{~b}$, does respond well to tactile stimulation, and a systematic representation of the contralateral body surface in area 1 forms a mirror image of the representation in area $3 \mathrm{~b}$. In addition, a third representation of at least much of the contralateral body surface, including face, hand, and forearm, exists in area 2 in anthropoid primates (Pons et al., 1985). The areas 1-2 region in galagos are similar in location, architecture, and connections to a band of cortex along the caudal border of $\mathrm{S} 1$ in tree shrews, rats, and other studied mammals and thus, as for areas 3a and $3 b$ (S1), appear to be an early feature of cortical organization in the evolution of mammals. We have tentatively termed this strip of cortex in galagos area 1-2 (Fig. 1), as this area has features of both areas 1 and 2 of anthropoid primates. However, there is no evidence yet that area 1-2 of galagos includes two parallel strips that are homologous to areas 1 and 2 in anthropoid primates. Our current hypothesis, based on present evidence, is that a single area in most mammals, and in prosimian primates, subdivided to become two distinct areas with the evolution of anthropoid primates.

Galagos have additional somatosensory areas in the cortex of the upper bank of the lateral sulcus and the insula in the depths of the sulcus (Wu and Kaas, 2003). Two of these areas, the second somatosensory area, S2, and the parietal ventral area, PV, have been described in a number of nonprimate mammals, as well as in several species of monkeys (Coq et al., 2004; Disbrow et al., 2003; Krubitzer and Kaas, 1990) and humans (Eickhoff et al., 2007). Both S2 and PV represent the contralateral body surface from the head along the $3 \mathrm{~b}$ border to foot deep in the sulcus. The two representations mirror each other and depend on inputs from area $3 \mathrm{~b}$ for activation. As S2 and PV have been identified in a number of mammals, including opossums, these two areas have been retained in primates from early mammals (Kaas, 2007). Galagos have caudal and rostral divisions of the ventral somatosensory area (VS in Fig. 1) that are also found in monkeys and humans but have not been reported for non-primate mammals, raising the possibility that VSc and VSr are areas that evolved in early primates. Other areas in the cortex of the lateral sulcus have been defined in galagos by patterns of connections with S2 and PV, and these include a parietal rostral area and a retroinsular area (Wu and Kaas, 2003). The parietal rostral area is in the expected location of gustatory cortex (G? in the unfolded lateral sulcus in Fig. 1), a region that responds to taste

Prog Brain Res. Author manuscript; available in PMC 2013 October 01. 
stimuli, but has not been well defined in primates (Kaas et al., 2006). Parts of the insula are involved in nociception, and in humans, empathy for the pain of others (Keysers et al., 2010). The insula is greatly expanded in humans and is involved in mediating empathy, pleasant touch, and other socially relevant functions. Likely, the insula is one of the most changed regions of the human brain.

\section{Motor cortex}

Motor cortex in galagos (Fig. 1) includes a number of areas that are also found in other primates (Wu et al., 2000). A primary motor area, M1, just rostral to areas $3 \mathrm{a}$ and $3 \mathrm{~b}$ of somatosensory cortex, has been identified in all studied placental mammals, and thus has an ancient origin. In primates, M1 has a large region devoted to hand movements, and in some primates, M1 has distinct rostral and caudal divisions (Preuss et al., 1997). M1 is more specialized for movements of individual digits in macaque monkeys than it is in galagos, as M1 organization reflects types of use of the hand. Galagos and other primates also have dorsal and ventral premotor areas, PMD and PMV, a supplementary motor area (SMA), and caudal and rostral cingulate motor areas $(\mathrm{CMr}$ and $\mathrm{CMc})$. A dorsal premotor area has been found in rodents and tree shrews (Remple et al., 2007) that may correspond to either SMA or PMD of primates. PMV may have emerged with the first primates. Both PMD and PMV have functionally distinct subdivisions in macaque monkeys (Fujii et al., 2000; Geyer et al., 2000; Luppino et al., 1999), suggesting further evolution of premotor cortex. An anterior part of ventral premotor cortex that is involved with orofacial movements has been suggested to be a homologue of the much more extensive Broca's area of the left cerebral hemisphere in humans (Petrides et al., 2005). Finally, the frontal eye field (FEF) where electrical stimulation produces eye movements is an area that has been identified in galagos, new and old world monkeys, and humans. The eye movements are evoked via connections with the superior colliculus and brainstem motor centers, but galagos appear to differ from monkeys in having very few projections from the FEF to the superior colliculus. As cortex rostral to M1 has few if any projections to the superior colliculus in most non-primate mammals, the sparseness or lack of such a projection in galagos may reflect the primitive condition.

\section{Posterior parietal sensorimotor cortex}

The organization of posterior parietal cortex is variable across primate taxa. Compared to rodents and tree shrews with very little cortex that can be considered posterior parietal cortex, all primates have a large posterior parietal region that is especially enlarged in humans. In galagos, PPC can be divided into two large regions, a posterior division with inputs from a collection of higher visual areas, and a rostral division with inputs from higher order somatosensory areas (Fig. 1). The caudal division of PPC gets inputs mainly from visual areas MT, MST, MTc, and DM, areas considered to be components of the dorsal stream of visual processing that mediate visuomotor guidance via connections with PPC (Goodale and Milner, 1992; Ungerleider and Mishkin, 1982). Visual inputs to rostral PPC largely depend on projections from caudal PPC to rostral PPC. Electrical stimulation of rostral, but not caudal, PPC evokes complex movements that depend on connections with motor and premotor cortex (Kaas et al., 2011). The types of movements evoked by electrical stimulation of rostral PPC depend on the location of the stimulating electrode so that hindlimb movements are evoked from the most medial sites, forelimb movements from more lateral sites, and face and eye movements from the most lateral sites. Even from anesthetized galagos, functionally meaningful complex behaviors can be evoked when electrical stimulation continues for the duration of the behavior (about $0.5 \mathrm{~s}$ ). Thus, reaching, defensive movements of the arm to protect the face and grasping movements can be evoked from a caudorostral sequence of locations in or near PPC (locations R, D, and G in Fig. 1). Other locations produce face, eye, and other movements. Note especially that grasping 
movements were evoked from a location that was largely in areas $1-2$, providing evidence that part of areas 1-2 is functionally related to PPC. A similar arrangement of reach, defense, and grasp zones exist in new world monkeys and in macaque monkeys (Gharbawie et al., 2011; Kaas et al., 2011), with the difference that these zones have a more rostrocaudal arrangement, especially in macaques, and these zones have more direct visual inputs from dorsal stream visual areas in macaques. Overall, the differences suggest that most of PPC is occupied by an expanded rostral division of PPC in anthropoid primates and that visual inputs to this expanded rostral division have become more direct and more important.

As the grasp zone in macaque monkeys is largely in area 2 and the grasp zone in new world monkeys is in cortex immediately caudal to area 1, it appears that new world monkeys have an area 2 that is separated from area 1, something that has been questioned. However, the location of the grasp zone in areas 1-2 in galagos suggests that galagos do not have separate areas 1 and 2 .

PPC in humans is a greatly expanded part of neocortex (Hill et al., 2010) and it contains subdivisions that may be homologous to those in monkeys, as well as those that may have been elaborated or developed in the ancestors of modern humans, allowing new abilities such as the extensive use of tools and the use of gestures for communication (Frey, 2007). A number of human abilities likely depend on PPC.

\section{Prefrontal cortex}

Prefrontal cortex of primates is a large division of neocortex that is thought to be especially important in mediating cognitive and social aspects of human behavior. Most mammals have an orbital region with lateral and medial components, as indicated for galagos in Fig. 1. There is also a granular frontal region rostral to the FEF that is less expansive in galagos than in anthropoid primates (Preuss and Goldman-Rakic, 1991), and possibly absent in mammals other than primates (Preuss, 1995). Many of the higher cognitive and social abilities of humans are attributed to frontal cortex, especially granular frontal cortex, and frontal cortex is larger in humans than in other primates. However, frontal cortex as a whole is not proportionately larger in humans than expected for a primate brain (Semendeferi et al., 2002). The functional organization of prefrontal cortex likely differs considerably across primate taxa, with evolved elaborations and multiple subdivisions in the human brain.

\section{The evolution of structural and cellular differences in cortical areas in primates}

Primate brains of all sizes differ from rodent brains, and likely all other mammals, in having more densely packed and overall smaller neurons, and this largely reflects the smaller neurons of neocortex in primates (Herculano-Houzel et al., 2007). In addition, neuron sizes and densities vary across cortical areas within primate species, and across primate taxa for homologous areas (Collins et al., 2010). When the densities of neurons were compared across the cortical sheet in galagos, new world monkeys, and old world macaques and baboons, neuron densities across cortical areas varied the least in galagos and the most in the larger brains of macaques and baboons. Even greater variability might be expected in human brains. Overall, the highest densities were observed in primary visual cortex across these primates, while secondary visual cortex and primary somatosensory cortex also had higher density values in the old world anthropoids. Lower cell densities were found in association areas with larger neurons. High densities of small neurons are useful in areas that need to segregate many inputs during processing for an analysis of details, while areas with large neurons of low densities are more useful for summing inputs for a more global analysis.

As galagos appear to have the fewest cortical areas, with macaques having more, and humans likely having many more, primates with large brains and many cortical areas have 
the advantage of being able to specialize some areas for analyzing local detail and others for integrative processing. Primates with fewer cortical areas need to preserve the more general functions of areas, and thus areas are less specialized and have less variability in neuron densities across areas. In large-brained primates with more cortical areas, we can expect some of these areas to become highly specialized, because other areas can be retained for a broad range of functions. But, such specializations come at a greater cost in smallbrained mammals with few cortical areas, since this limits options.

As a clear exception, present-day tarsiers are such highly specialized visual predators that they eat only small invertebrates and vertebrates, and no plants. The ability to be an effective, nocturnal visual predator depends on a highly specialized visual system (Collins et al., 2005; Wong and Kaas, 2010). In tarsiers, a single visual area, V1, occupies over $20 \%$ of neocortex, more than in any other primate, and V1 is more distinctly divided into layers and sublayers than in any other primate (Fig. 2). The large V1 with densely packed small neurons preserves the detail of central vision, so that tarsiers can detect small cryptic prey. The distinctive lamination of V1, reminiscent of the laminated optic tectum of birds of prey, reflects the specializations of cellular morphologies of different input and output layers and sublayers for different functional roles in tarsiers.

\section{Epilogue}

Given the limitations of space and present understandings, this review only touched on the huge topic of the evolution of neocortex in primates. Here, we tried to reconstruct from comparative and fossil evidence the organization of the neocortex of the common primate ancestor of all extant primates. We know that primates emerged over 80 million years ago as a branch of the Euarchontoglire superclade (Murphy et al., 2004). This branch included several lines of archaic primates that became extinct, and the stem euprimates that led to the present-day galagos, lorises, tarsiers, and the greatly varied anthropoid monkeys, apes, and hominids (humans and extinct species more closely related to us than chimpanzees). The closest living relatives of primates are the Scandentia (tree shrews) and Dermoptera (flying lemurs) of the Archontan branch of Euarchontoglires. The more distant Glires branch includes rodents and lagomorphs. The extinct archaic primates had smaller brains with less neocortex than modern primates, and it is difficult to deduce how their cortex was organized. Perhaps the most we can do is compare the cortical organization of extant primates with the most primitive cortical features to the cortical features of tree shrews and rodents, as flying lemurs are not available for experimental study. When we do that, we can surmise that the proportionately smaller neocortex of archaic primates had less posterior parietal, frontal, and temporal cortex, and that the ventral visual stream for object vision via temporal cortex and the dorsal visual stream for visually guided motor behavior were less developed than in any extant primates. Additionally, the premotor areas of frontal cortex were likely less developed. Thus, motor abilities and motor flexibility would have been less pronounced in archaic primates. Finally, a less expansive prefrontal cortex would suggest that archaic primate behavior was more dependent on ongoing sensory events, and less on social experience and previous environmental events.

In contrast to these uncertain possibilities, comparative studies of cortical organization in members of extant primate taxa are now extensive enough to extract many shared features of cortical organization that likely reflect those retained from a common stem euprimate ancestor. It is likely that all of the cortical areas illustrated for extant galagos (Fig. 1) were present in that common ancestor, as these areas and subdivisions of areas can be identified in other primates. However, this proposal is incomplete, perhaps in need of correction, and it should be evaluated further in ongoing studies of cortical organization in prosimians and other branches of the primate radiation. Cortical organization in galagos needs further study, especially in temporal, posterior parietal, prefrontal, and medial wall regions. Nevertheless, 
the evidence to date suggests that prosimian galagos present a very good model of what the neocortex of early euprimates was like, not only in terms of areal organization but also in terms of structural and connectional organization.

What is more difficult to deduce and fully describe is how neocortex became modified in the many branches of the primate radiation. Here, we barely touched on this important topic. The challenge is great given the many primate species, the difficulty or impossibility of conducting experimental studies on many of these species, and the major gaps in the radiation produced by extinctions. While modern humans and chimpanzees are separated from a common ancestor by only a few million years, our brains are three times larger, with most of this increase over the past 2 million years of hominin evolution. Only relatively recently, within thousands of years, we have become the only surviving species within the formerly varied hominin branch. This loss, plus the limited ways in which the brains of apes and humans can be studied, makes it very difficult to reconstruct the evolution of the human brain, although information on how neocortex is organized in humans is being rapidly acquired. As fMRI, optical imaging and other recent technical advances have greatly magnified what can be learned about brain organization, a much better understanding of the evolution of cortical organization in primates can be expected in the near future.

\section{References}

Allman JM, Kaas JH. A representation of a visual field in the caudal third of the middle temporal gyrus of the owl monkey (Aotus trivirgatus). Brain Research. 1971; 31:85-105. [PubMed: 4998922]

Allman JM, Kaas JH. A crescent-shaped cortical visual area surrounding the middle temporal area (MT) in the owl monkey (Aotus trivirgatus). Brain Research. 1974; 81:199-213. [PubMed: 4215542]

Allman JM, Kaas JH. The dorsomedial cortical visual area: A third tier area in the occipital lobe of the owl monkey (Aotus trivirgatus). Brain Research. 1975; 100:473-487. [PubMed: 811327]

Avzevedo FAC, Carvalho LRB, Grinberg LT, Farfel JM, Ferretti REJ, Leite REP, et al. Equal numbers of neuronal and nonneuronal cells make the human brain an isometrically scaled-up primate brain. The Journal of Comparative Neurology. 2009; 513:532-541. [PubMed: 19226510]

Brugge, JF. Auditory areas in primates. In: Woolsey, CN., editor. Cortical sensory organization. Clifton, NJ: Humana Press; 1982. p. 59-70.

Casagrande, VA.; Kaas, JH. The afferent, intrinsic, and efferent connections of primary visual cortex in primates. In: Peters, A.; Rockland, K., editors. Cerebral cortex. Vol. 10. New York: Plenum Press; 1994. p. 201-259.

Collins CE, Airey DC, Young NA, Leitch DB, Kaas JH. Neuron densities vary across and within cortical areas in primates. Proceedings of the National Academy of Sciences USA. 2010; 107(36): 15927-15932.

Collins CE, Hendrickson A, Kaas JH. Overview of the visual system of Tarsius. The Anatomical Record Part A. 2005; 287(1):1013-1025.

Collins CE, Stepniewska I, Kaas JH. Topographic patterns of V2 cortical connections in a prosimian primate (Galago garnetti). The Journal of Comparative Neurology. 2001; 431:155-167. [PubMed: 11169997]

Coq JO, Qi HX, Collins CE, Kaas JH. Anatomical and functional organization of somatosensory areas of the lateral fissure of the New World titi monkey (Callicebus moloch). The Journal of Comparative Neurology. 2004; 476(4):363-387. [PubMed: 15282711]

Disbrow E, Litinas E, Recanzone GH, Padberg J, Krubitzer L. Cortical connections of the second somatosensory area and the parietal ventral area in macaque monkeys. The Journal of Comparative Neurology. 2003; 462:382-399. [PubMed: 12811808]

Eickhoff SB, Grefkes C, Zilles K, Fink GR. The somatotopic organization of cytoarchitectonic areas on the human parietal operculum. Cerebral Cortex. 2007; 17:1800-1811. [PubMed: 17032710] 
Felleman DJ, Nelson RJ, Sur M, Kaas JH. Representations of the body surface in areas 3b and 1 of postcentral parietal cortex of Cebus monkeys. Brain Research. 1983; 268(1):15-26. [PubMed: 6860957]

Frey, SH. Neurological specializations for processing faces and objects. In: Kaas, JH.; Preuss, TM., editors. Evolution of nervous systems. Vol. 4. Oxford; Academic Press; 2007. p. 395-406.

Fujii N, Mushiake H, Tanji J. Rostrocaudal distinction of the dorsal premotor area based on oculomotor involvement. Journal of Neurophysiology. 2000; 83:1764-1769. Rapid Communication. [PubMed: 10712497]

Geyer S, Zilles K, Luppino G, Matelli M. Neurofilament protein distribution in the macaque monkey dorsolateral premotor cortex. European Journal of Neuroscience. 2000; 12:1554-1566. [PubMed: 10792433]

Gharbawie OA, Stepniewska I, Qi HX, Kaas JH. Multiple parietal-frontal networks mediate grasping in macaque monkeys. The Journal of Neuroscience. 2011; 31:11660-11677. [PubMed: 21832196]

Goodale MA, Milner AD. Separate visual pathways for perception and action. Trends in Neuroscience. 1992; 15:20-25.

Herculano-Houzel S, Collins CE, Wong P, Kaas JH. Cellular scaling rules for primate brains. Proceedings of the National Academy of Sciences of the United States of America. 2007; 104:3562-3567. [PubMed: 17360682]

Hill J, Inder T, Neil J, Dierker DL, Harwell J, Van Essen D. Similar patterns of cortical expansion during human development and evolution. Cerebral Cortex. 2010; 8(3):13135-13140.

Kaas JH. What, if anything, is SI? The organization of "first somatosensory area" of cortex. Physiological Review. 1983; 63:206-231.

Kaas, JH. The evolution of visual cortex in primates. In: Kremers, J., editor. The structure, function and evolution of the primate visual system. Chichester, UK: John Wiley and Sons; 2005. p. 267-283.

Kaas, JH. The evolution of sensory and motor systems in primates. In: Kaas, JH.; Preuss, TM., editors. Evolution of nervous systems. Vol. 4. London: Academic Press; 2007. p. 35-57.

Kaas, JH. The evolution of auditory cortex: The core areas. In: Winer, JA.; Schreiner, CE., editors. The auditory cortex. New York: Springer-Verlag; 2011. p. 407-427.

Kaas JH, Gharbawie OA, Stepniewska I. The organization and evolution of dorsal stream multisensory motor pathways in primates. Frontiers in Neuroanatomy. 2011; 5(34):1-7. [PubMed: 21373368]

Kaas JH, Hackett TA. Subdivisions of auditory cortex and processing streams in primates. Proceedings of the National Academy of Sciences of the United States of America. 2000; 97:11793-11799. [PubMed: 11050211]

Kaas JH, Morel A. Connections of visual areas of the upper temporal lobe of owl monkeys: The MT crescent and dorsal and ventral subdivisions of FST. The Journal of Neuroscience. 1993; 3:534546. [PubMed: 8381166]

Kaas, JH.; Preuss, TM. Archontan affinities as reflected in the visual system. In: Szalay, F.; Novacek, M.; McKenna, M., editors. Mammalian phylogeny. New York: Springer-Verlag; 1993. p. 115-128.

Kaas JH, Qi HX, Iyengar S. Cortical network for representing the teeth and tongue in primates. The Anatomical Record. 2006; 288A:182-190. [PubMed: 16411246]

Kaskan PM, Dillenburger BC, Lu HD, Roe AW, Kaas JH. Orientation and direction-of-motion response in the middle temporal visual area (MT) of New World Owl Monkeys as revealed by intrinsic-signal optical imaging. Frontiers in Neuroanatomy. 2010; 4:23. [PubMed: 20661299]

Keysers C, Kaas JH, Gazzola V. Somatosensation in social perception. Nature Reviews. Neuroscience. 2010; 11(6):417-428. [PubMed: 20445542]

Krubitzer LA, Kaas JH. The organization and connections of somatosensory cortex in marmosets. The Journal of Neuroscience. 1990; 10(3):952-974. [PubMed: 2108231]

Luppino G, Muvata A, Govoni P, Matelli M. Largely segregated parietofrontal connections linking rostral intraparietal cortex (areas AIP and VIP) and the ventral premotor cortex (areas F5 and F4). Experimental Brain Research. 1999; 128:181-187.

Lyon DC, Kaas JH. Connectional evidence for dorsal and ventral V3, and other extrastriate areas in the prosimian primate, Galago garnetti. Brain, Behavior and Evolution. 2002a; 59:114-129. 
Lyon DC, Kaas JH. Evidence for a modified V3 with dorsal and ventral halves in macaque monkeys. Neuron. 2002b; 33:453-461. [PubMed: 11832231]

Lyon DC, Kaas JH. Evidence from V1 connections for both dorsal and ventral subdivisions of V3 in three species of New World monkeys. The Journal of Comparative Neurology. 2002c; 449:281297. [PubMed: 12115680]

Martin RD. Palaeontology: Chinese lantern for early primates. Nature. 2004; 427:22-23. [PubMed: 14702069]

Murphy WJ, Pevzner PA, O'Brien JO. Mammalian phylogenomics comes of age. Trends in Genetics. 2004; 20(12):631-639. [PubMed: 15522459]

Ni X, Wang Y, Hu Y, Li C. A euprimate skull from the early Eocene of China. Nature. 2003; 427:6568. [PubMed: 14702085]

Petrides M, Cadoret G, Mackey S. Orofacial somatomotor responses in the macaque monkey homologue of Broca's area. Nature. 2005; 435:1235-1238. [PubMed: 15988526]

Pons TP, Garraghty PE, Cusick CG, Kaas JH. The somatotopic organization of area 2 in macaque monkeys. The Journal of Comparative Neurology. 1985; 241:445-466. [PubMed: 4078042]

Poremba A, Mishkin M. Exploring the extent and function of higher-order auditory cortex in rhesus monkeys. Hearing Research. 2007; 229:14-23. [PubMed: 17321703]

Preuss TM. Do rats have prefrontal cortex? The Rose-Woolsey-Akert program reconsidered. Journal of Cognitive Neuroscience. 1995; 7:1-24. [PubMed: 23961750]

Preuss TM, Beck PD, Kaas JH. Areal, modular, and connectional organization of visual cortex in a prosimian primate, the slow loris (Nycticebus coucang). Brain, Behavior and Evolution. 1993; 42(6):321-335.

Preuss TM, Goldman-Rakic PS. Architectonics of the parietal and temporal association cortex in the strepsirhine primate Galago compared to the anthropoid primate Macaca. The Journal of Comparative Neurology. 1991; 310:475-506. [PubMed: 1939733]

Preuss TM, Kaas JH. Cytochrome oxidase 'blobs'; and other characteristics of primary visual cortex in a lemuroid primate, Cheirogaleus medius. Brain, Behavior and Evolution. 1996; 47(2):103-112.

Preuss TM, Stepniewska I, Jain N, Kaas JH. Multiple divisions of macaque precentral motor cortex identified with neurofilament antibody SMI-32. Brain Research. 1997; 767:148-153. [PubMed: 9365028]

Remple MS, Reed JL, Stepniewska I, Lyon DC, Kaas JH. The organization of frontoparietal cortex in the tree shrew (Tupaia belangeri): II Connectional evidence for a frontal-posterior parietal network. The Journal of Comparative Neurology. 2007; 501:121-149. [PubMed: 17206607]

Rosa MGP, Casagrande VA, Preuss TM, Kaas JH. Visual field representation in striate and prostriate cortices of a prosimian primate (Galago garnetti). Journal of Neurophysiology. 1997; 77:31933217. [PubMed: 9212268]

Rosa MGP, Krubitzer LA. The evolution of visual cortex: Where is V2? Trends in Neuroscience. 1999; 22:242-248.

Scott SK, Johnsrude IS. The neuroanatomical and functional organization of speech perception. Trends in Neuroscience. 2003; 26:100-107.

Semendeferi K, Lu A, Schenker N, Damasio H. Humans and great apes share a large frontal cortex. Nature. 2002; 5:272-276. On-line (February, 2002).

Sweet RA, Dorph-Petersen KA, Lewis DA. Mapping auditory core, lateral belt, and parabelt cortices in the human superior temporal gyrus. The Journal of Comparative Neurology. 2005; 491:270289. [PubMed: 16134138]

Tootell RB, Taylor JB. Anatomical evidence for MT and additional cortical visual areas in humans. Cerebral Cortex. 1995; 5(1):39-55. [PubMed: 7719129]

Ungerleider, LG.; Mishkin, M. Two cortical visual systems. In: Ingle, DG.; Goodale, MA.; Mansfield, RJQ., editors. Analysis of visual behavior. Cambridge: MIT Press; 1982. p. 549-586.

Van Hooser SD, Heimel JA, Chung S, Nelson SB. Lack of patchy horizontal connectivity in primary visual cortex of a mammal without orientation maps. Journal of Neuroscience. 2006; 26:7680 7692. [PubMed: 16855096] 
Wong P, Collins CE, Kaas JH. Overview of sensory systems of Tarsius. International Journal of Primatology. 2010; 31:1002-1031.

Wong P, Kaas JH. Architectonic subdivisions of neocortex in the Galago (Otolemur garnetti). Anatomical Record. 2010; 293(6):1033-1069.

Woods DL, Herron TJ, Cate AD, Yund EW, Stecker GC, Rinne T, et al. Functional properties of human auditory cortical fields. Frontiers in Systems Neuroscience. 2010; 4:1-13. [PubMed: 20204156]

Wu CWH, Bichot NP, Kaas JH. Converging evidence from microstimulation, architecture, and connections for multiple motor areas in the frontal and cingulate cortex of prosimian primates. The Journal of Comparative Neurology. 2000; 423:140-177. [PubMed: 10861543]

$\mathrm{Wu} \mathrm{CW}$, Kaas JH. Somatosensory cortex of prosimian galagos: Physiological recording, cytoarchitecture, and corticocortical connections of anterior parietal cortex and cortex of the lateral sulcus. The Journal of Comparative Neurology. 2003; 457(3):263-292. [PubMed: 12541310]

Prog Brain Res. Author manuscript; available in PMC 2013 October 01. 


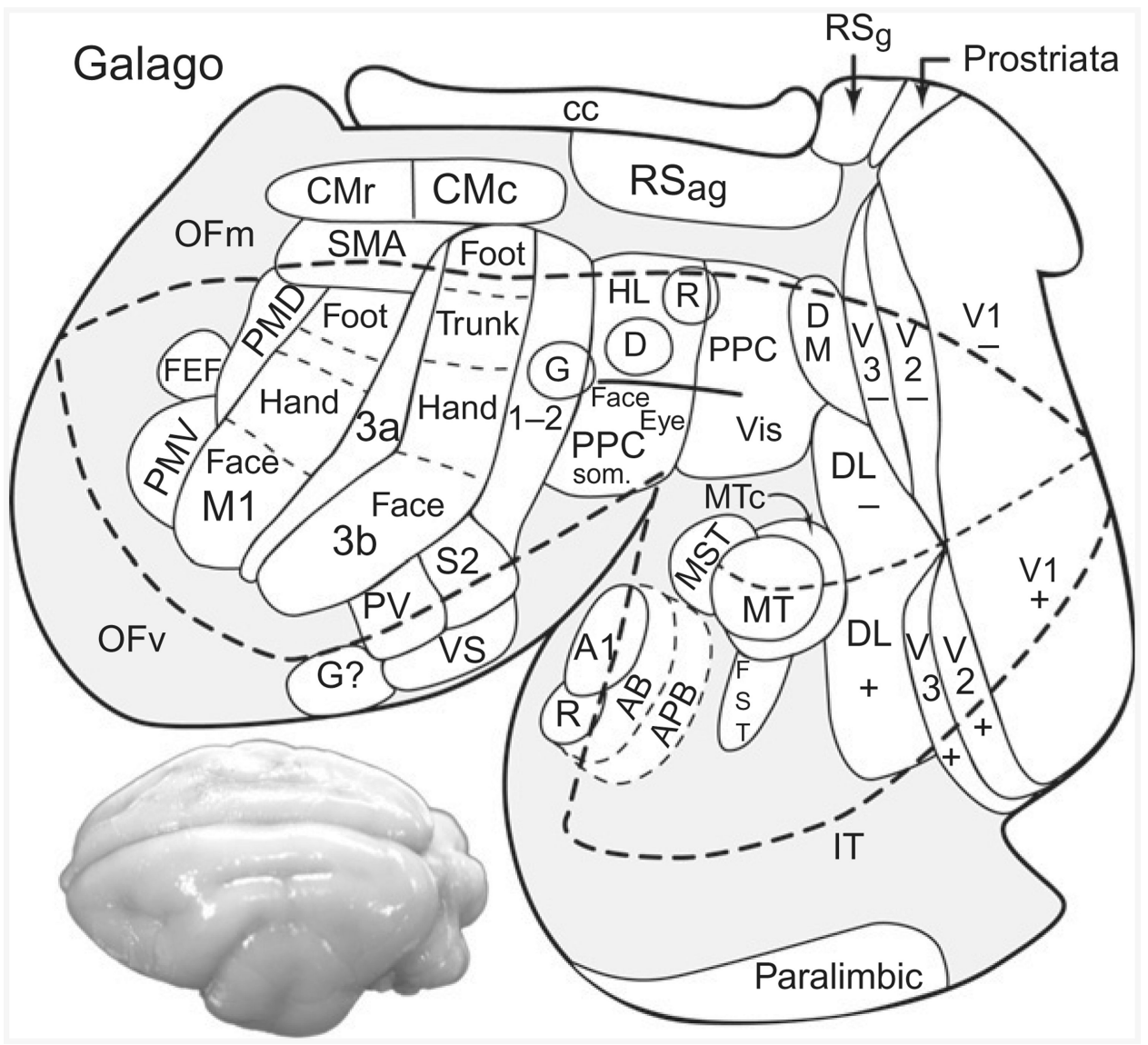

Fig. 1.

Areal subdivisions of neocortex in a prosimian primate, galago (Otolemur garnetti). For orientation, a dorsolateral view of the brain is on the lower left. The larger figure is of the neocortex after it has been removed from the rest of the brain, fissures opened, and flattened so that all of the cortical surface can be seen, and cortical areas can be depicted relative to each other. Visual areas include primate visual cortex (V1), the second visual area (V2), the third visual area (V3), the dorsomedial visual area (DM), the dorsolateral visual area (DL, also known as V4), the middle temporal visual area (MT), the MT crescent (MTc), the middle superior temporal area (MST), and the fundal area of the superior temporal sulcus (FST) which has dorsal and ventral subdivisions. Inferior temporal cortex (IT) contains several visual areas, but they have not been well defined. Auditory cortex includes a core of two primary areas, primary auditory cortex (A1) and the rostral auditory area (R), as well as a surrounding belt of as many as eight secondary areas (AB) and an adjoining auditory parabelt (APB) with two major divisions. Somatosensory cortex includes a primary area (3b or S1), a proprioceptive area (3a), a secondary area caudal to S1 (areas 1-2), a second area (S2), a parietal ventral area (PV), a ventral somatosensory area (VS), possibly a gustatory (taste) area (G?), and other less well-defined areas in insular cortex. Motor cortex includes a primary area (M1), a ventral premotor area (PMV), a dorsal premotor area (PMD), a supplementary motor area (SMA), a frontal eye field (FEF), and rostral (CMr) and caudal $(\mathrm{CMc})$ cingulate motor areas. Posterior parietal cortex (PPC) has a large caudal division with visual inputs (Vis), and a large rostral division with somatosensory inputs (Som.) and a somatotopic organization from hindlimb (HL) to face and eye. Territories within PPC are indicated where reaching $(\mathrm{R})$ or defensive $(\mathrm{D})$ movements can be evoked with electrical stimulation. A territory for grasping $(\mathrm{G})$ movements is marked in areas $1-2$. Foot $(F)$ and 
hand $(\mathrm{H})$ regions in M1 are marked and representations of upper (+) and lower (-) visual field representation are indicated for some visual areas. Retrosplenial agranular (RSag) and retrosplenial granular (RSg) areas are marked. The thicker dotted line outlines the extent of neocortex visible on a lateral view of the intact brain, the dotted line through visual areas indicates the location of the representation of the zero horizontal meridian, and dashed lines in $\mathrm{M} 1$ and 3b delimit the representation segments for major body parts. Medial (OFm) and ventral (OFv) orbital-frontal regions are marked. Corpus callosum (CC) is marked. 


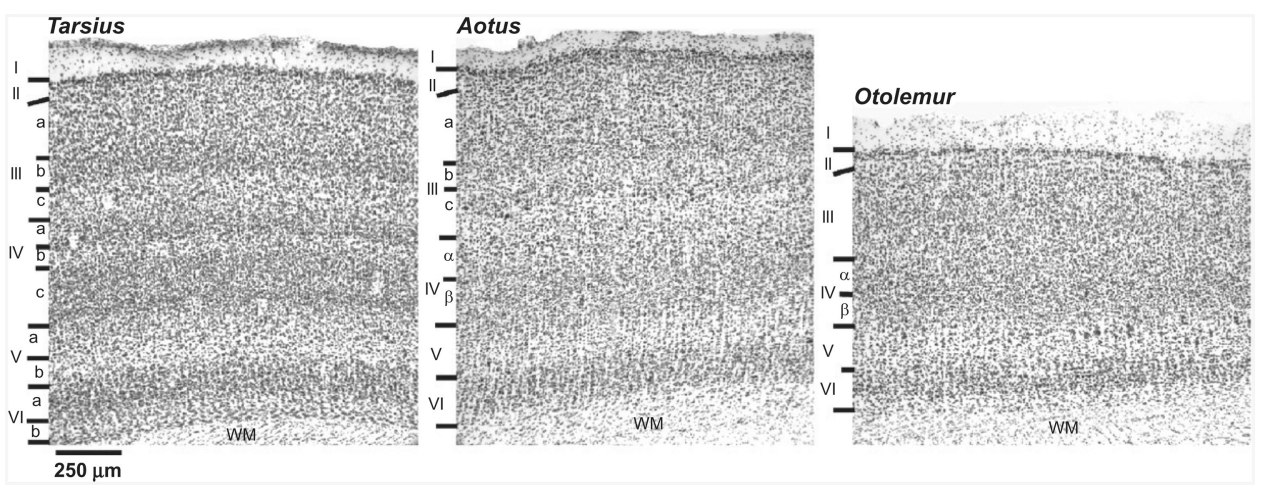

Fig. 2.

Nissl-stained sections from primary visual cortex (V1) of Tarsius and Otolemur (galago) and Aotus (a nocturnal new world monkey) for comparison. Note the more obvious layering and sublayering of V1 in Tarsius, less in Aotus, and the least in Otolemur. WM, white matter.

Roman numbers mark the six cortical layers, while letters are used for sublayers. See Collins et al. (2005) for a full description of the tarsier visual system. 